\title{
Design of decentralized load-frequency controllers for interconnected power systems with AC-DC parallel tie-lines and GDB nonlinearity using MOEA

\author{
S. Ganapathy and S. Velusami
}

Dept. of Electrical Engineering, Annamalai University, Annamalainagar-608 002, Tamilnadu, India

ganapathy1967@gmail.com; profvels@yahoo.co.in

Abstract: A new design of decentralized load-frequency controllers for interconnected power systems with AC-DC parallel tie-lines and governor dead band (GDB) nonlinearity using multi-objective evolutionary algorithm (MOEA) is proposed. A HVDC link is connected in parallel with an existing ac tie-line to stabilize the frequency oscillations of the ac system. The proposed control strategy is mainly based on a compromise between integral squared error and maximum stability margin criteria. A two area interconnected thermal power system is considered to demonstrate the validity of the proposed controller. The simulation results show that the system dynamic performance has been improved with increased stability margin when compared to a conventional controller.

Keywords: AC-DC tie-lines, governor dead band nonlinearity, load-frequency control, multi-objective evolutionary algorithm.

Introduction

The interconnected power system presents a great challenge in power system design and operation. The load-frequency control (LFC) problem has gained much importance because of the size and complexity of modern interconnected power systems. The objective of LFC is to regulate the output powers of regulating plants so that the frequency of power system and tie-line powers are kept within prescribed limits. Many control strategies for LFC of power systems have been proposed and investigated by many researchers over the past several years (Shayeghi et al., 2009).

Majority of the works carried out earlier is centered on interconnected power systems considering the area interconnection with ac tie-lines only. However, there has been a tremendous growth of the HVDC transmission system due to economic, environmental and performance advantages over the other alternatives. Hence, it has been applied widely in operating a dc link in parallel with an ac link interconnecting control areas to get an improved system dynamic performance with greater stability margins under small disturbances in the system (Kumar \& Ibraheem, 2004). Only scantly information s available on LFC of interconnected power systems connected via HVDC link in parallel with ac link. Therefore, this paper considers LFC of an interconnected power system with a dc tie-line in parallel with an ac tieline. Incremental dc power flow is considered as an additional state variable in the LFC strategy.

In most of the LFC studies, the effect of the Governor dead band (GDB) nonlinearity is neglected for simplicity. But for a realistic analysis of the performance of the system, this should be included as it has a considerable effect on the amplitude and settling time of the oscillations (Tripathy et al., 1982). The effect of GDB nonlinearity is considered in this study, by describing function approach in the state space model.

The optimum parameter values of the conventional proportional plus integral (PI) controller have been obtained in the literature by minimizing the popular integral of the squared error (ISE) criterion (Elgerd and Fosha, 1970). Controllers designed on the basis of ISE criterion are often of practical significance because of the minimization of control effort. But the system has poor relative stability. Hence, to obtain the decentralized controllers with improved stability margin, they are designed on the basis of maximum stability margin (MSM) criterion using Lyapunov method. However, controllers designed on the basis of MSM criterion do not posses the inherent good properties of the controller designed on the basis of ISE criterion even though there is improvement in stability (Chidambaram \& Velusami, 2005). Therefore, it is expected that an appropriate multiobjective control strategy will be able to give a better solution for this problem.

Many evolutionary techniques have been extensively used for the design of LFC for isolated as well as interconnected power systems (Mathur \& Ghosh, 2006). But, they have been mainly applied to LFC problems treated as single-objective optimization problems.

Hence, a new design of proportional plus integral controllers using multi-objective evolutionary algorithm (MOEA) is proposed in this work, for the decentralized load-frequency control of interconnected power systems with ac-dc parallel tie-lines and including GDB nonlinearity, to achieve a better transient, as well as steady state response and closed loop stability of the system. The LFC problem is formulated as a multiobjective optimization problem where ISE criterion and MSM criterion are treated as competing objectives. The proposed controller has been applied to an interconnected two-area thermal power system with acdc parallel tie-lines. Each area has two thermal generating units and includes GDB nonlinearity.

\section{Statement of the problem}

The block diagram representation of a two area interconnected thermal power system with ac-dc parallel tie-lines is shown in Fig.1. Each of the area in the interconnected power system consists of two thermal generating units and includes GDB nonlinearity. GDB is defined as the total magnitude of a sustained speed change within which there is no change in valve position. A describing function approach is used to incorporate the GDB nonlinearity (Tripathy et al., 1982).

The dynamic behaviour of the LFC system is described by the state space equation:
Research article

CIndian Society for Education and Environment (iSee)
"Power system"

http://www.indjst.org
Ganapathy \& Velusami Indian J.Sci.Technol. 
$\dot{x}=A x+B u+\Gamma d$ where

$x=\left[\Delta F_{1} \Delta P_{g 11} \Delta X_{e 11}^{\prime} \Delta P_{g 12} \Delta P_{g 12}^{\prime} \Delta X_{e 12}^{\prime} \Delta P_{e 1}\right.$

$\left.\Delta P_{d c e 1} \Delta F_{2} \Delta P_{g 21} \Delta X_{e 21}^{\prime} \Delta P_{g 22} \Delta P_{g 22}^{\prime} \Delta X_{e 22}^{\prime}\right]^{\top}$

$\boldsymbol{u}=\left[\Delta P_{c 1} \Delta P_{c 2}\right]^{\mathrm{T}}$

$\boldsymbol{d}=\left[\begin{array}{ll}\Delta P_{d 1} & \Delta P_{d 2}\end{array}\right]^{\mathrm{T}}$

are the state, control and disturbance vectors and $A, B$ and $\Gamma$ are respectively system state matrix, control input matrix and disturbance input matrix of appropriate dimensions. The corresponding co-efficient matrices are obtained using the nominal system parameter values given in Appendix. A step load disturbance of $1 \%$ has been considered as a disturbance in the system.

It is known that, by incorporating an integral controller, the steady state requirements can be achieved. In order to introduce integral function in the controller, the system equation (1) is augmented with new state variables defined as the integral of $\mathrm{ACE}_{\mathrm{i}}\left(\int_{\mathrm{i}} \mathrm{dt}\right), \mathrm{i}=1,2$.

The augmented system of the order $(2+n)$ may be described as
Vol.2 No. 7 (July 2009)

ISSN: 0974- 6846

$$
\begin{aligned}
\dot{\bar{X}} & =\bar{A} \bar{x}+\bar{B} u+\bar{\Gamma} d \\
\bar{x} & \left.=\left[\begin{array}{c}
\int \boldsymbol{v} \boldsymbol{d t} \\
\boldsymbol{x}
\end{array}\right]\right\} 2 \\
\overline{\boldsymbol{A}} & =\left[\begin{array}{ll}
0 & C \\
0 & A
\end{array}\right] \bar{B}=\left[\begin{array}{l}
0 \\
B
\end{array}\right] \text { and } \bar{\Gamma}=\left[\begin{array}{l}
0 \\
\Gamma
\end{array}\right]
\end{aligned}
$$

The decentralized feedback control law may be written in terms of $\boldsymbol{v}_{i}$ as:

$$
\boldsymbol{u}_{\boldsymbol{i}}=-\boldsymbol{k}_{\boldsymbol{i} 1} \int \boldsymbol{v}_{\boldsymbol{i}} \boldsymbol{d t}-\boldsymbol{k}_{i 2} \boldsymbol{v}_{i}, i=1,2
$$

where $\boldsymbol{k}_{i}^{T}=\left[\begin{array}{ll}k_{i 1} & k_{i 2}\end{array}\right]$ is a 2-dimensional integral and proportional feedback gain vector (Ramar \& Velusami, 1989).

This design assumes that, the two area interconnected power system consists of 2 - identical areas. Therefore, the decentralized integral feedback gains $\left(k_{1 I}=k_{2 l}=k_{I}\right)$ and the decentralized proportional controller feedback gains $\left(k_{12}=k_{22}=k_{P}\right)$ of the 2 - identical areas are assumed to be equal.

Fig. 1. Block diagram representation of a two area interconnected thermal power system with ac-dc parallel tie-lines and GDB nonlinearity

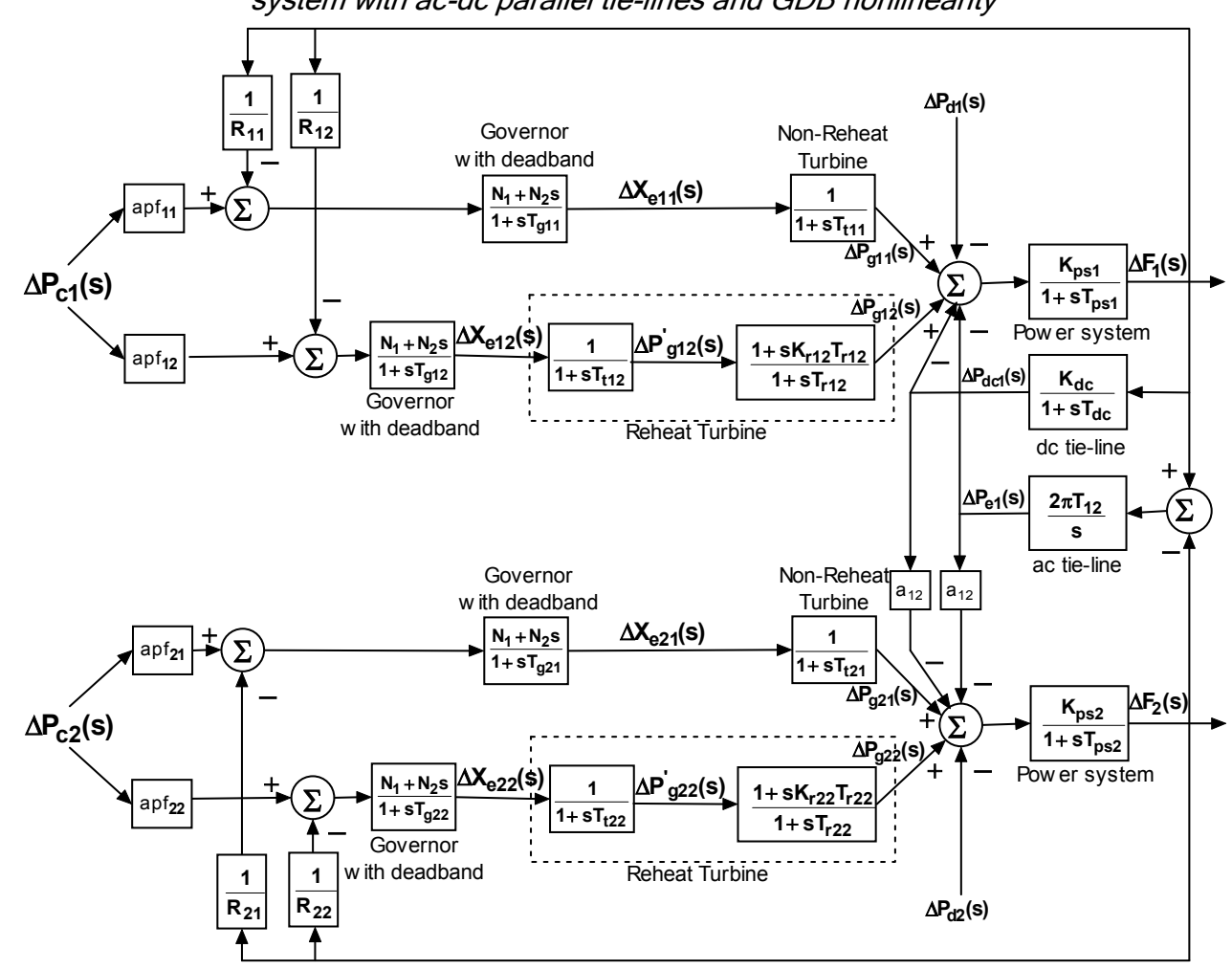

Research article

CIndian Society for Education and Environment (iSee)
Ganapathy \& Velusami Indian J.Sci.Technol. 
Design of decentralized proportional plus integral controller using ISE criterion

The objective is to obtain the optimum values of the controller parameters that minimize the performance index,

$$
\boldsymbol{J}_{i}=\int_{0}^{t}\left(\boldsymbol{x}_{e i}^{T} \boldsymbol{W}_{i} \boldsymbol{x}_{e i}\right) d t, i=1,2
$$

where

$$
W_{i}=\operatorname{diag}\left\{w_{i 1} w_{i 2} w_{i 3}\right\} \text { and } x_{e i}{ }^{T}=\left[\begin{array}{ll}
\Delta f_{i} \Delta p_{e i} & \Delta p_{d c e i}
\end{array}\right]
$$

$w_{i 1}, w_{i 2}$ and $w_{i 3}$ are weighting factors for the frequency deviation, ac tie-line power deviation and dc tie-line power deviation respectively of area $\mathrm{i}$ and are chosen as unity. The decentralized proportional plus integral controller gains using ISE criterion are designed as discussed in (Nanda and Kaul, 1978) and the values obtained are $k_{P}=0.33$ and $k_{l}=0.34$.

Design of decentralized proportional plus integral controller using MSM criterion

The controller designed on the basis of Integral Squared Error criterion tends to show a rapid decrease in the large initial error. Hence, the response is fast and oscillatory. Thus, the system has poor relative stability (Ogatta, 1970). Therefore, the design of proportional plus integral controller with improved stability using MSM criterion by Lyapunov method (Tripathy et al., 1982) is discussed in this section.

The stability index to be minimized is

$$
\eta=\bar{x}^{T} P \bar{x}
$$

Where $P$ is a symmetric positive definite matrix obtained from the solution of

$$
\hat{A} P+P \hat{A}=-Q
$$

where $Q$ is a positive semi-definite matrix and $\hat{A}$ is augmented system matrix. The weighting matrix $Q$ is chosen as

$$
\boldsymbol{Q}=\operatorname{diag}\{0,0,1,0,0,0,0,0,1,1,1,0,0,0,0,0\} \text {. }
$$

The proportional controller feedback gain $k_{P}^{\prime}$, corresponding to minimum value of stability index $\eta$, is obtained using the MSM criterion by plotting the stability curve for various values of $k_{P}^{\prime}$ against the stability index $\eta$ (Tripathy et al.,1982). The integral feedback gain $k_{\prime}^{\prime}$ is treated as zero throughout in this design. From the stability curve, the optimal proportional controller feedback gain $k_{P}^{\prime}=-0.1$ is obtained. Next, the stability curve for various values of $k_{I}^{\prime}$ is obtained by simulating the closed loop system and keeping $k_{P}^{\prime}=k_{p}^{\prime}(o p t)$. From the curve, the optimal integral controller feedback gain $k_{\prime}^{\prime}=1.1$ is obtained.

Design of proposed decentralized proportional plus integral controller

Controllers designed on the basis of MSM criterion do not posses the inherent good properties of the controller designed on the basis of ISE criterion except for improvement in stability. Therefore, a new controller

Research article

CIndian Society for Education and Environment (iSee) design needs to be developed based on a compromise between the ISE design criterion and MSM design criterion in order to obtain satisfactory closed loop system performance and stability (Chidambaram \& Velusami, 2005).

An attempt has been made in this section to design a decentralized controller using Multi-Objective Evolutionary Algorithm.

\section{Multi-Objective Evolutionary Algorithm}

Multi-objective optimization methods deal with finding optimal solutions to problems having multiple objectives. These objectives often conflict each other so that improving one of them will deteriorate another objective function. Therefore, the solution to a Multi-objective optimization problem is normally not a single value but instead a set of values called the "Pareto-Optimal Set". No solution from this set of optimal solution can be said to be better than another solution. This procedure is practical because the user gets an opportunity to investigate a number of other trade-off solutions before choosing one particular optimal solution.

A Multi-objective optimization problem can be mathematically defined as (Deb, 2001):

Minimize/ Maximize

$$
f_{m}(X), \quad m=1,2, \ldots M \text {; }
$$

Subject to $\mathrm{J}$ inequality constraints

$$
\begin{aligned}
& g_{j}(X) \geq 0, \quad j=1,2, \ldots J ; \\
& \text { and K equality constaints } \\
& h_{k}(X)=0, \quad k=1,2, \ldots K ; \\
& \mathrm{x}_{\mathrm{i}}(\mathrm{L}) \leq \mathrm{x}_{\mathrm{i}} \leq \mathrm{x}_{\mathrm{i}}(\mathrm{U}), \quad i=1,2, \ldots . . n .
\end{aligned}
$$

The last set of constraints are called variable bounds, restricting each decision variable $x_{i}$ to take a value within a lower $x_{i}^{(L)}$ and an upper $x_{i}^{(U)}$ bound.

There are $M$ objective functions $f(X)=\left(f_{1}(X), f_{2}(X), \ldots . f_{M}(X)\right)^{T}$ considered in the above formulation.

A solution $X$ is a vector of $n$ decision variables. $X=\left(\mathrm{x}_{1}, \mathrm{x}_{2}, \ldots . . . \mathrm{x}_{\mathrm{n}}\right)^{\top}$.

Evolutionary algorithms (EAs) are a natural choice for solving multi-criterion optimization problems because of their population-based nature. A number of Paretooptimal solutions can, in principle, be captured in an EA population, thereby allowing a user to find multiple Pareto-optimal solutions in one simulation run. Different approaches of MOEA have been used by different researchers for multi-objective optimization, each one having its merits and demerits. Among various MOEAs, $\varepsilon$ - MOEA has shown the best performance.

Hence, in this study, a steady state multi-objective evolutionary algorithm based on $\varepsilon$-dominance concept is used (Deb et al., 2003).

Here, two populations (EA and archive) are evolved simultaneously and independently. Using one solution each from both populations, two off-spring solutions are created through mating. Each off-spring is then used to update both parent and archive populations. The archive 
population is based on the $\varepsilon$-dominance whereas a usual dominance concept is used to update the present population. The final archive members after a specified number of iterations are reported as the obtained solutions (Deb et al., 2003). The algorithm for $\varepsilon$ - MOEA is given in (Shin, et al., 2006).

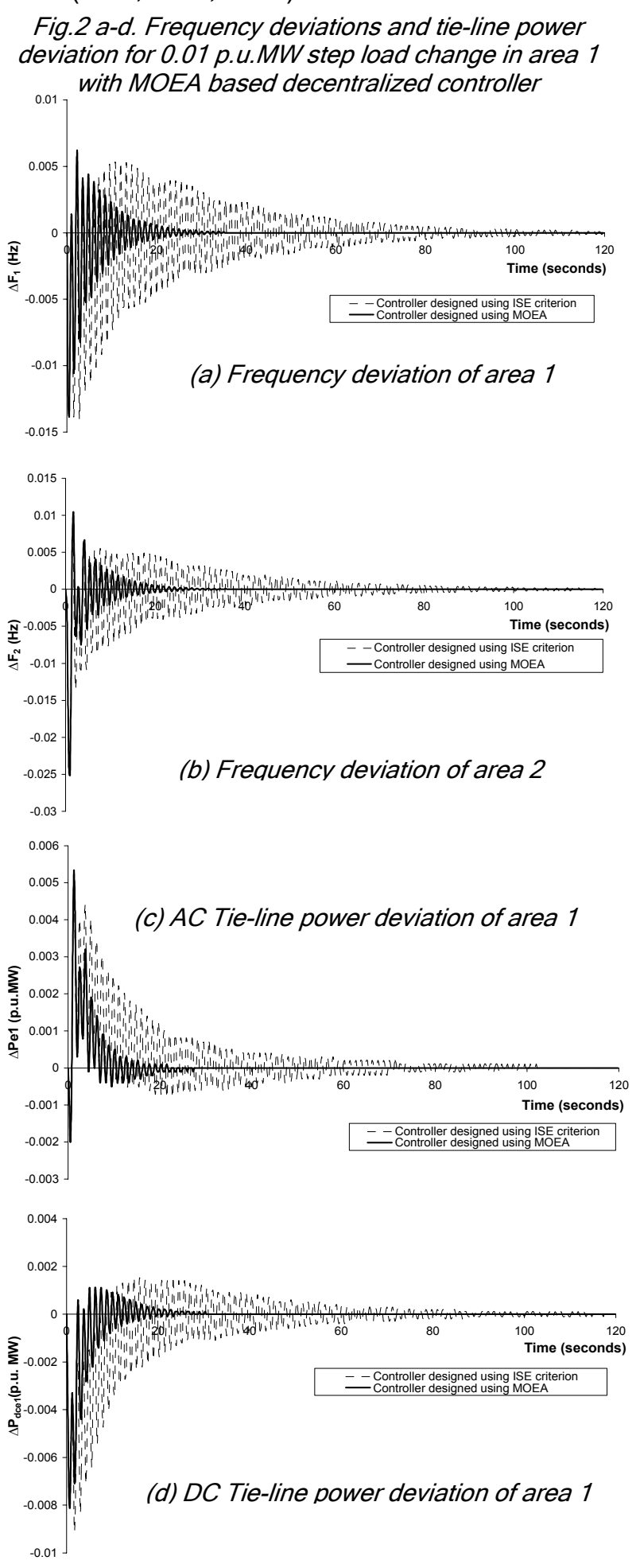

Design of proposed decentralized proportional plus integral controller using MOEA

An attempt has been made in this section, to apply MOEA to the LFC problem with ISE criterion and MSM criterion as conflicting objectives.

The LFC problem can be formulated as

Minimize

Subject to

$$
\begin{aligned}
& f_{1}(X)=f_{1}\left(x_{1}, x_{2}\right)=f_{1}\left(k_{P m}, k_{I m}\right)=J_{1} \\
& f_{2}(X)=f_{2}\left(x_{1}, x_{2}\right)=f_{2}\left(k_{P m}, k_{I m}\right)=\eta
\end{aligned}
$$

$$
\begin{aligned}
& k_{P m}{ }^{(L)} \leq k_{P_{m}} \leq k_{P_{m}}{ }^{(U)} \\
& k_{I m}{ }^{(L)} \leq k_{I m} \leq k_{I m}{ }^{(U)}
\end{aligned}
$$

The proportional controller feedback gains obtained by ISE and MSM criteria, namely $k_{P}=0.33$ and $k_{P}^{\prime}=-0.1$, are treated as the upper and lower bounds for the decision variable $k_{P m}$ in the MOEA. Similarly, the integral controller feedback gains obtained by MSM criterion and ISE criterion, namely, $k_{\prime}^{\prime}=1.1$ and $k_{l}=0.34$, are treated as the upper and lower bounds for the decision variable $k_{/ m}$. The proposed controller feedback gains are obtained as $k_{P m}=0.14$ and $k_{I m}=0.96$ using MOEA. This design ensures that, the controller feedback gains will always be within the ranges of the gains obtained from the ISE criterion and the MSM criterion. Therefore, the controller will guarantee the stability. Further the controller possesses improved stability when compared to the controller obtained using ISE criterion. The overall performance of these controllers will be better than that of the controller designed on the basis of MSM criterion. The choice of $\varepsilon$-MOEA parameters was done according to general guidelines available in the literature. A population size of 100, the real-parameter Simulated Binary Crossover (SBX) recombination operator with a crossover probability of 1 and a distribution index of 15 for crossover, and a polynomial mutation operator with a mutation probability of $1 / n$ ( $n=$ number of decision variables) and a distribution index of 20 for mutation, have been used. The recommended values of $\varepsilon_{1}=0.05$ and $\varepsilon_{2}=0.05$ are found to be robust enough and are used in our study.

\section{Simulation results and observations}

The decentralized controller with output feedback is designed using MOEA with multiple objectives, namely the ISE criterion and MSM criterion, and implemented in the interconnected two-area thermal power system with ac-dc parallel tie-lines and GDB nonlinearity. The system is simulated with the proposed controller for 0.01 p.u.MW step load change in area 1 and the corresponding frequency deviation $\Delta f$ and tie-line power deviation $\Delta \mathrm{P}_{\mathrm{e} 1}$ are plotted with respect to time. For easy comparison, the responses of $\Delta f$ and $\Delta \mathrm{P}_{\mathrm{e} 1}$ of the system are shown along with the responses obtained with the 
optimal decentralized proportional plus integral controller designed on the basis of ISE criterion in Fig. 2.

It is observed that the proposed controller offers better dynamic performance and any further improvement in one of the design objectives will lead to degradation in the other objective.

The cost function values of both the controllers are given in Table 1. It is observed from Fig. 2 \& Table 1 that the proposed controller using MOEA has improved stability as well as reduced cost function value when compared to the controller designed on the basis of ISE criterion.

Table1. Comparison of cost function values
\begin{tabular}{|l|c|c|}
\hline $\begin{array}{l}\text { Type of proportional } \\
\text { plus integral controller }\end{array}$ & $\begin{array}{c}\text { Feedback } \\
\text { gains }\end{array}$ & $\begin{array}{c}\text { cost function } \\
\text { value }\end{array}$ \\
\hline $\begin{array}{l}\text { Controller designed } \\
\text { using ISE criterion }\end{array}$ & $k_{P}=0.33$ & 0.4352 \\
$k_{/}=0.34$ & \\
\hline $\begin{array}{l}\text { Controller designed } \\
\text { using MOEA }\end{array}$ & $k_{P m}=0.14$ & 0.1230 \\
$k_{/ m}=0.96$ & \\
\hline
\end{tabular}

\section{Conclusion}

A design of multi-objective evolutionary algorithm based decentralized load-frequency controllers for an interconnected power system with ac-dc parallel tie-lines is presented. This design has been successfully applied to an interconnected two-area thermal power system with two thermal generating units and Governor dead band in each area. A comparative simulation study of the proposed controller with conventional controller shows that the proposed controller can yield a better dynamic performance with good closed-loop stability of the system following a step load change.

Appendix

Data for the interconnected two-area thermal power system:

Rating of each area $=2000 \mathrm{MW}$,

Base power $=2000 \mathrm{MVA}$,

$f^{\circ}=60 \mathrm{~Hz}, a_{12}=-1$,

$\mathrm{R}_{11}=\mathrm{R}_{12}=\mathrm{R}_{21}=\mathrm{R}_{22}=2.4 \mathrm{~Hz} /$ p.u. $\mathrm{Hz}$,

$\mathrm{T}_{\mathrm{G} 11}=\mathrm{T}_{\mathrm{G} 12}=\mathrm{T}_{\mathrm{G} 21}=\mathrm{T}_{\mathrm{G} 22}=0.08 \mathrm{~s}$,

$\mathrm{T}_{\mathrm{t} 11}=\mathrm{T}_{\mathrm{t} 12}=\mathrm{Tt}_{21}=\mathrm{Tt}_{21}=0.3 \mathrm{~s}$,

$\mathrm{T}_{\mathrm{r} 12}=\mathrm{T}_{\mathrm{r} 22}=10 \mathrm{~s}, \mathrm{~K}_{\mathrm{ps} 1}=\mathrm{K}_{\mathrm{ps} 2}=120 \mathrm{~Hz} /$ p.u.MW,

$\mathrm{K}_{\mathrm{r} 12}=\mathrm{K}_{\mathrm{r} 22}=0.5, \mathrm{~T}_{\mathrm{ps} 1}=\mathrm{T}_{\mathrm{ps} 2}=20 \mathrm{~s}$,

$\beta_{1}=\beta_{2}=0.425$ p.u. $\mathrm{MW} / \mathrm{Hz}$,

$2 \pi \mathrm{T}_{12}=0.545$ p.u. $\mathrm{MW} / \mathrm{Hz}$

$\Delta \mathrm{P}_{\mathrm{d} 1}=0.01$ p.u.MW,

$\mathrm{apf}_{11}=\mathrm{apf}_{12}=\mathrm{apf}_{21}=\mathrm{apf}_{22}=0.5$

Data for dc link

$\mathrm{K}_{\mathrm{dc}}=1.0, \mathrm{~T}_{\mathrm{dc}}=0.5 \mathrm{~s}$.

All notations carry the usual meanings.
Vol.2 No. 7 (July 2009)

ISSN: 0974- 6846

\section{References}

1. Chidambaram IA and Velusami S (2005) Design of decentralized biased controllers for load-frequency control of interconnected power systems. Electric Power Components \& Systems. 33, 1313-1331.

2. Deb K (2001) Multi-objective optimization using evolutionary algorithms, John Wiley, UK.

3. Deb K, Mohan M and Mishra S (2003) A fast multiobjective evolutionary algorithm for finding wellspread pareto-optimal solutions. kanGAL Technical Report No. 2003002. Kanpur Genetic Algorithms Laboratory. Indian Institute of Technology. Kanpur, India, pp: 1-18.

4. Elgerd OE and Fosha CE (1970) Optimum megawatt-frequency control of multi area electric energy systems. IEEE Transactions on Power Apparatus \& Systems. 89(2), 556-563.

5. Kumar P and Ibraheem (2004) Study of dynamic performance of power systems with asynchronous tie-lines considering parameter uncertainties. Journal of Institution of Engineers (India): Electrical Engineering Division. 8, 35-42.

6. Mathur HD and Ghosh S (2006) A comprehensive analysis of intelligent controllers for load frequency control. Proceedings of IEEE Power India Conference. New Delhi, India, pp: 853-857.

7. Nanda J and Kaul BL (1978) Automatic generation control of an interconnected power system. IEE Proceedings. 125(5), 385-390.

8. Ogatta K (1970), Modern control engineering. Prentice-Hall.New Jersey.

9. Ramar K and Velusami S (1989) Design of decentralized load-frequency controllers using pole placement technique. Electric Machines \& Power Systems. 16, 193-207.

10. Shayeghi H, Shayanfar HA and Jalili A (2009) Load frequency control strategies: A state-of-the-art survey for the researcher. Energy Conversion \& Management. 50(2), 344-353.

11. Shin SY, Lee IH and Zhang BT (2006) Microarray probe design using $\varepsilon$-multi-objective evolutionary algorithms with thermodynamic criteria. Proc. EvoWorkshops2006. LNCS 3907. Budapest. Hungary, pp: 184-195.

12. Tripathy SC, Hope GS and Malik OP (1982) Optimisation of load-frequency control parameters for power systems with reheat steam turbines and governor dead band nonlinearity. IEE Proc. 129 (1), 10-11.
"Power system"

http://www.indjst.org
Ganapathy \& Velusami Indian J.Sci.Technol. 